\title{
Multi-beam coverage and beamforming technology for high altitude platform station communication system
}

\author{
Mingxiang Guan ${ }^{*}$, Zhou Wu, Yingjie Cui, Xuemei Cao, Le Wang, Jianfeng Ye and Bao Peng
}

\begin{abstract}
Multi-beam antenna technology can effectively mitigate the impact of deep fading caused by multipath propagation on communication quality through spatial diversity and increase the reliability of wireless mobile communication systems. At the same time, the multi-beam antenna with beamforming algorithm as the key technology can realize spatial division multiple access (SDMA) by automatically adapting the weight vector of the antenna array, enhance the expected value, and suppress the interference. A novel beamforming technology increases the inner circle beam width and reduces the outer ring beam width to minimize the difference between the internal and external cell link SNR. This study considers reducing the number of cells by reasonably allocating beams, which satisfies both the requirements of coverage link quality and the difficulty of forming antenna beams. This research subject applies the scheme and strategy of improving multi-beam antenna technology in the same system. Under the requirement of cell coverage and partitioning, the related technologies and strategies combining multi-beamforming technology and space-time block code technology can obtain high coding gain and diversity gain without sacrificing bandwidth and effectivelyx improve the wireless systematic capacity.
\end{abstract}

Keywords: HAPS communication, Multi-beam, Beamforming, Coverage

\section{Introduction}

Interference in high altitude platform station (HAPS) communication is another major factor affecting and limiting system performance in addition to channel fading. It includes both inter-symbol interference (ISI) due to signal multipath propagation and delay spread and co-channel interference (CCI) and adjacent channel interference within the network. These interferences will cause problems such as crosstalk, dropped calls, or reduced communication quality during communication, thus reducing user satisfaction with the service. In addition, the existence of these interferences also limits the reuse of the system frequency domain, time domain, and code domain resources, which greatly hinders the improvement of system capacity. Multi-beam smart antenna technology is an effective technical means to solve these problems [1].

* Correspondence: guanmx@sziit.edu.cn

School of Electronic Communication Technology, Shenzhen Institute of Information Technology, Shenzhen, China
The multi-beam antenna technology is based on the fact that the wireless signal is spatially separable. First, an antenna array composed of a plurality of array elements is used, and secondly, an advanced signal processing algorithm is used to weight each antenna element, so that the pattern of the antenna array form a high gain in the desired direction to enhance the desired signal. In the interference direction, a null steering is formed to suppress the interference signal, thereby effectively improving the signal-interference-noise ratio (SINR) of the receiving end, thereby improving system performance and increasing system capacity.

The application of multi-beam antenna forming techniques in HAPS communication can bring the following benefits:

(1). Improve spectral efficiency and increase system capacity [2]: Space division multiple access is achieved by using spatial characteristics at the same time interval, the same frequency and the same 
code channel, thereby improving system capacity and spectrum utilization efficiency.

(2). Reduce system interference [3]: By shaped beam, the beam null steering is aligned with the interference signal direction. Thus, the interference can be effectively suppressed.

(3). Reduce system construction costs [4]: Since the multi-beam antenna technology can expand the coverage area, the number of high-altitude platforms can be relatively reduced. In addition, the antenna characteristics of the multi-beam antenna can be adjusted with the help of software to make the application more flexible. These methods have reduced the construction cost of the network to some extent.

It is well known that in the actual wireless mobile communication process, the existence of multipath fading and various interferences (including co-channel interference, adjacent channel interference, or external interference), limited spectrum resources and increasing demand for data transmission rate are simultaneously existing. If you simply use multi-beam antenna technology, it is difficult to meet actual needs in many cases [5]. Therefore, research on the schemes and strategies for improving multi-beam antenna technology in the same system becomes more and more important.

This topic attempts to do some work in this area, the key point is to study the related technologies and strategies of multi-beamforming technology and space-time block code technology under the premise of considering cell coverage and partitioning requirements. Space-time block code technology can achieve high coding gain and diversity gain without sacrificing bandwidth [6]. On the condition of a relatively simple receiver structure, the space-time structure of the space-time block code can effectively improve the capacity of the wireless system [7]. However, the space-time block code brings significant inter-symbol interference and inter-user interference to some extent while bringing significant diversity gain. The reduction of inter-symbol interference and the suppression of multi-user interference are typical advantages of multi-beamforming techniques. Therefore, it is easy to consider combining the two strategies so that the two strategies can complement each other and meet the needs of the development of the HAPS communication system.

Naguib and Calderbank [8] propose a method for joint beamforming in uplink space-time block codes. Its basic idea is to use a smart antenna array for beamforming on the platform receiver side and use space-time coding on the MS transmitter side.

This method is really very effective in uplink wireless transmission. However, it cannot be directly ported to the downlink because the antenna array cannot be used due to size limitations on the mobile station side. In addition, it cannot bear the complexity burden of beamforming. In the HAPS communication system, the downlink will carry more multimedia services, requiring higher system capacity and communication quality. Therefore, it is more necessary to study the beamforming technology applied to the downlink at the high-altitude platform.

Zhu and Lim and $\mathrm{Na}$ et al. $[9,10]$ propose a spacetime block code joint beamforming technique based on polarized antennas for downlink applications.

The basic idea of this type of joint technology is to use the different polarizations of the antennas to achieve the irrelevant channel, apply space-time block coding, and then perform beamforming in each polarization direction. The advantage of this method is that the structure is simple, the antenna array element spacing can be half wavelength, and the beams of the desired user are formed in each polarization direction. However, its disadvantage is that system performance is greatly affected by polarization cross-polarization discrimination (XPD). When the XPD value is high, the correlation between the two polarized channels increases, and the effect of space-time coding will decrease, which will bring higher false detection probability to the receiver decoding.

Guan et al. [11] propose a technique for space-time block code joint beamforming based on sub-array diversity structure for downlink. This type of technology generally assumes that the space-time encoder outputs $\mathrm{N}$ sub-streams and sends them to $\mathrm{N}$ antenna sub-arrays. The channels between each sub-array and the receiver antenna are mutually uncorrelated so that it could meet the irrelevant channel requirements of space-time code transmission. Within each sub-array, the channel between all antenna elements and the receiver antenna is highly correlated, and beamforming can be used to obtain the array gain.

Simple structure and lower requirements on the receiver are the advantage of this solution. However, due to the requirement of irrelevance between the channels formed by each sub-array and the receiving end, the spacing between the sub-arrays should be far enough, which actually greatly increases the size of the platform antenna array. In addition, each sub-array is a function of beamforming independently. Therefore, each subarray needs to be configured with enough array elements. This has led to a greater demand for the number of highaltitude platform-end antenna elements, which are difficult to achieve due to limited loads at high-altitude platform.

Guan and Wang [12] propose a downlink space-time block code joint beamforming technique using a single antenna array. The basic idea of the technique is to obtain two parallel weighting vectors simultaneously 
applied to the same transmit antenna array by eigendecomposing the correlation matrix of the channel. The two parallel weighting vectors can be implemented simultaneously: first, it is the primary purpose to ensure that the two weighted signals arriving at the receiver are uncorrelated, and secondly, the weights could be filtered on the basis of satisfying the first purpose, and then getting the highest possible SNR at the receiving end.

Compared with the previous scheme, using a single antenna array to reduce the size of the antenna at the platform end is the advantage of this scheme. In this way, the two transmit beams of the user are completed by using the same antenna array, and it could realize the sharing of the antenna elements between the two beams. Therefore, the requirement for the total number of antenna elements of the platform can be reduced. Zhu extended this scheme in the literature [13-15]; he uses two inter-related sub-antenna arrays for transmission; and although the performance is improved, it needs to increase the number of array elements in the antenna array. These programs can still only be applied to singleuser situations.

In addition, $\mathrm{Na}$ et al. $[16,17]$ analyze the effect of different angular spreads on the performance of space-time coding combining beamforming transmission strategies. Research shows that beamforming plays a dominant role when the angular spread is small, and space-time coding plays an absolute role when the angle spread is large. Dammann et al. [18] study the performance and algorithm of space-time coding joint beamforming in frequency selective fading channels.

A novel beamforming technology increases the inner circle beam width and reduces the outer ring beam width to minimize the difference between the internal and external cell link SNR in the study. This study considers reducing the number of cells by reasonably allocating beams, which satisfies both the requirements of coverage link quality and the difficulty of forming antenna beams. This research subject applies the scheme and strategy of improving multi-beam antenna technology in the same system. Under the requirement of cell coverage and partitioning, the related technologies and strategies combining multi-beamforming technology and space-time block code technology can obtain high coding gain and diversity gain without sacrificing bandwidth and effectively improve the wireless systematic capacity.

\section{Methodology}

The topology of the antenna array formed by the transmit beam of the multi-beam antenna system may be uniform linear, circular, planar, and so on. Its typical feature is that all array elements of the entire antenna array are related in terms of spatial characteristics and the geometrical structure is that the spacing between the antenna elements is very close. The number of array elements of the transmitting antenna array is $M$, and the array meets the following requirements: (1) the spacing of the array elements is small enough to ensure that the amplitudes of the signals received by the array elements are the same, generally $\lambda / 2, \lambda$ is the carrier wavelength; (2) there is no coupling between the array elements; (3) the incident signal band on the array aperture is small relative to the carrier frequency. $\theta_{0}$ is the desired user direction. $\theta_{i}$ is the number $i$ interfering user direction, and $W=\left[w_{0}, w_{1}, \ldots, w_{M-1}\right]$ is the weight vector of the antenna array at the transmitting end.

Assuming that both the desired user and the interfering user are in the far area of the antenna array and without considering the receiver noise and assuming that the transmit power of each transmit antenna is constant, the electric field strength observed for the farfield receiving point in direction $\theta$ can be expressed as

$$
E(\theta)=\sum_{i=0}^{M-1}|s| \alpha_{m} w_{m}^{*} e^{j \pi m \sin \theta}
$$

A $m$ is the wave attenuation factor from the transmitting element $m$ to the receiving point in the far zone. " $a$ " means doing the conjugate operation to the complex number $a$. Assuming that the receiving point is in the far area of the antenna array and the distance is much larger than the aperture of the antenna array, it can be considered that the wave attenuation factor $\alpha_{m}$ of all antenna elements reaches the same receiving point, that is, $\alpha_{m} \approx \alpha_{0}$. Therefore, the above formula can be rewritten as

$$
E(\theta)==\alpha_{0}|s| \mathbf{W}^{H} \mathbf{a}(\theta)
$$

$\boldsymbol{a}(\theta)=\left[1, e^{j \pi \sin \theta}, e^{j 2 \pi \sin \theta}, \ldots e^{j(M-1) \pi \sin \theta}\right]^{T}$ is the steering vector of the array in the $\theta$ direction. The normalized electric field strength in the $\theta$ direction can be expressed as

$$
F(\theta)=\left|\frac{E(\theta)}{\alpha_{0}|s|}\right|=\left|\mathbf{W}^{H} \mathbf{a}(\theta)\right|
$$

It can be seen from Eq. (3) that the beam pattern formed by the transmit beam is exactly the same as the beam pattern formed by the receive beam, so many optimal weighting coefficient optimization criteria for receive beamforming can be applied in transmit beamforming. However, it is also recognized that there are some differences between transmit beamforming and receive beamforming. These differences are mainly manifested in the following:

(1). Receive beamforming can easily obtain accurate uplink channel characteristic inbeam-forming, but it 
is difficult to know the characteristic inbeamforming of the downlink channel. There are currently three ways to solve this problem. The first method is to use the uplink channel inbeamforming to estimate the downlink channel, which is feasible in TDD (time division duplex) system. The second method is designed for the FDD (frequency division duplex) system, which is used to estimate the uplink channel inbeam-forming after being subjected to a frequency correction. The third method is to make a closed-loop structure.

However, the last solution is not optimistic, since it will waste system resources, additional delays, and vulnerability to upstream channel interference.

(2). The transmit beamforming is completed at the transmitting end and only acts on the transmitted signal without amplifying the receiver noise. Therefore, when studying the transmit beamforming, the influence of the beamforming algorithm on the received noise can be ignored.

(3). Transmit beamforming should ensure a constant total transmit power. For wireless communication systems, the total transmit power of the system is limited, thus the transmit beamforming must ensure that the transmit power of the system is not increased.

Thereby, the transmit beamforming can be mapped to the following optimization problems:

$$
\begin{aligned}
J=\max _{\mathbf{w}}\left|\mathbf{W}^{H} \mathbf{a}\left(\theta_{0}\right)\right|^{2} & \\
\text { Constraint } 1 \mathbf{W}^{H} \mathbf{a}\left(\theta_{i}\right) & =0, \quad \begin{array}{c}
i \\
\end{array} \\
& =1,2, \ldots(N-1)
\end{aligned}
$$

\section{Constraint $2 \quad \mathbf{W}^{H} \mathbf{W}=1$}

$\theta_{0}$ is the desired user direction. $\theta_{i}$ is the direction of the number $i$ interference user, and the system includes $N$ users. The objective function is to ensure that the main lobe is formed in the desired user direction. The first constraint is to form the null steering in the direction of other $N-1$ interfering users, thereby reducing interference to other users. The second constraint is to ensure that the total transmit power of the system is constant.

\section{Beamforming research of HAPS communication multi-beam antenna system}

In order to solve this optimization problem, it is assumed that the base station transmitter knows the downlink signal wave off-angle inbeam-forming. The solution is solved by a slight improvement of the max SNR method and the linear constrained minimum variance (LCMV) method in the received beamforming. These methods are physically designed to achieve the maximum "expectation/interference" ratio, so the optimal solution they can achieve should be equivalent.

Here, another method for determining the transmit beamforming weight vector based on zero space is also given.

From the first constraint of Eq. (4), it can be seen that the weight vector $W$ of the $M \times 1$ dimension must be within the zero space of the $(N-1) \times M$ dimension matrix $B=\left[a\left(\theta_{1}\right), a\left(\theta_{2}\right), \ldots a\left(\theta_{N-1}\right)\right]^{H}$. The weight vector $W$ is decomposed into the product of a $M \times(M-L)$ dimensional matrix and a $(M-L) \times 1$ dimensional column vector, that is, $\boldsymbol{W}=\boldsymbol{P q}$, where $L$ is the rank of the matrix $\boldsymbol{B}$, that is, $L=\operatorname{rank}(\boldsymbol{B})$. Singular value decomposition of the matrix $\boldsymbol{B}$

$$
\mathbf{B}=\mathbf{U} \boldsymbol{\Sigma}\left[\begin{array}{ll}
\mathbf{V}^{(1)} & \mathbf{V}^{(0)}
\end{array}\right]^{H}
$$

$\Sigma$ is the diagonal matrix composed of the singular values of matrix $\boldsymbol{B} . \boldsymbol{V}^{(1)}, \boldsymbol{V}^{(0)}$ are the right singular vectors corresponding to non-zero singular values and zero singular values, respectively. According to the nature of matrix singular value decomposition, when $\boldsymbol{P}=\boldsymbol{V}^{(0)}$, it can be guaranteed that the weight vector $W$ is in the zero space of the $\boldsymbol{B}$ matrix, that is, the first constraint in Eq. (4) is satisfied.

Bring the above results into the objective function of the optimization problem, we could get

$$
\begin{aligned}
J & =\max _{\mathbf{W}}\left\{\mathbf{W}^{H} \mathbf{a}\left(\theta_{0}\right) \mathbf{a}^{H}\left(\theta_{0}\right) \mathbf{W}\right\} \\
& =\max _{\mathbf{W}}\left\{\mathbf{q}^{H} \mathbf{P}^{H} \mathbf{a}\left(\theta_{0}\right) \mathbf{a}^{H}\left(\theta_{0}\right) \mathbf{P q}\right\}
\end{aligned}
$$

Let $\boldsymbol{K}=\boldsymbol{P}^{\mathrm{H}} \boldsymbol{a}\left(\theta_{0}\right) \boldsymbol{a}^{\mathrm{H}}\left(\theta_{0}\right) \boldsymbol{P}$, for the abovementioned optimization problem, when $\boldsymbol{q}$ is the eigenvector corresponding to the maximum eigenvalue of the matrix $K$, the above formula can reach the maximum.

$$
\mathbf{K}=\left[\mathbf{v}_{1}, \mathbf{v}_{2}, \ldots \mathbf{v}_{M-L}\right] \boldsymbol{\Lambda}\left[\mathbf{v}_{1}, \mathbf{v}_{2}, \ldots \mathbf{v}_{M-L}\right]^{H}
$$

where $\Lambda$ is a diagonal matrix composed of matrix $K$ eigenvalues, and the eigenvalues are in descending order. $v_{1}, v_{2}, \ldots, v_{M-1}$ are eigenvectors corresponding to the eigenvalues in $\boldsymbol{\Lambda}$. Thus,

$$
\mathbf{q}=\mathbf{v}_{1}
$$

Finally, the weighting vector obtained by multiplying $\boldsymbol{q}$ and $\boldsymbol{P}=\boldsymbol{V}^{(\mathbf{0})}$ is normalized (the second constraint of Eq. (4) is satisfied, that is, the total transmit power is constant for each user), the final weight vector of the required transmit beamforming can be obtained, that is 


$$
\mathbf{W}=\frac{\mathbf{V}^{(0)} \mathbf{v}_{1}}{\left[\left(\mathbf{V}^{(0)} \mathbf{v}_{1}\right)^{H} \mathbf{V}^{(0)} \mathbf{v}_{1}\right]^{1 / 2}}
$$

In summary, it can be seen that the beamforming technology of the multi-beam antenna system, that is, the beamforming technique, utilizes the difference in the wavelength separation angle observed at the base station end during signal space transmission to achieve spatial differentiation. The direction of the formed beam is also measured by angle.

The organic combination of space-time block code technology and multi-beam antenna beamforming technology can effectively improve the communication quality and communication capacity of the entire communication system. However, there are huge contradictions between the two technologies on the requirements of the application environment, mainly in the requirements of the correlation of the different spatial channels formed by the array antennas of the transmitting antennas to the receiving antennas.

As can be seen from the description of the spacetime block code and the smart antenna beamforming technique in the previous section, the space-time block code requires that the fading channels formed from the respective transmit antenna elements to the receive antennas are mutually uncorrelated, that is, the interval between the transmitting antenna elements is required to be sufficiently large (about $10 \lambda$, $\lambda$ is the carrier wavelength). However, the smart antenna beamforming technique requires an antenna spacing that is sufficiently small (less than or equal to $\lambda / 2$ ) to enable antenna shaping using signal correlation. This contradiction determines that space-time block codes and smart antenna beamforming techniques cannot be directly applied in combination, and the special strategies must be adopted to solve the problem.

\section{The coverage characteristics of HAPS communication system}

At present, there are two common methods for cell division. The first method is to use the terrestrial cellular system for division. The coverage area is divided into a plurality of equal-sized equilateral hexagons, and each cell is covered by adjusting the shape of the antenna beam. The advantage of this kind of division is that each cell is equal in size and easy to manage; the disadvantage is that antenna beamforming is difficult, and beam-tobeam interference is easy to form. The second method is an elliptical cell structure. By fixing the size of the antenna beam, it is covered in an elliptical form. The antenna required for this structure is simple, but the single-beam coverage of the outer ring is much larger than that of the inner ring. Such excessive coverage may cause insufficient channel capacity. Obviously, a cell division method, which is suitable for HAPS communication, can be found which can combine the advantages of the above two cell division methods and appropriately avoid their disadvantages. And utilizing this method could avoid the problem of increased user switching and the change of a large number of cell positions caused by the platform swinging and rotating.

Compared with the cell scheme, the elliptic cell scheme has the problem of uneven SNR distribution and a large difference in the coverage area.

But the cell also has problems. The antenna beam is covered by the division of the cell, and the antenna beam is required to be elliptical. The smaller the elevation angle is, the greater the curvature is. Therefore, this method has strict requirements on the manufacturing technology of the antenna, which greatly increases the cost. Therefore, an improved multi-layer elliptical cell scheme can be considered. Increasing the width of the inner ring beam and reducing the width of the outer ring beam could minimize the difference in the SNR between the inner and outer cell links. At the same time, the area of the inner circle cell is increased, and the area of the outer circle cell is reduced. Besides, it is also considered to meet the requirements of the quality of the covered link by reasonably allocating the beam, and the difficulty of antenna beamforming should also be properly considered.

The choice of beam size needs to be optimized based on parameters such as capacity, number of antennas, and beamforming difficulty. While ensuring the number of beams, that is, the number of cells, increasing the spatial division multiplexing degree of the platform spectrum can increase the system capacity.

After adopting the structural design of the improved multi-layer elliptical cell, the ground coverage formed by the beam under the condition of cell swing and rotation is shown in Figs. 1 and 2.

As can be seen from Figs. 4 and 5, when the platform is swaying, the multi-beam antenna scans the ground to form a strip cover; when the platform rotates, the multibeam antenna scans the ground to form a ring cover. Regardless of whether it is a strip cover or a ring cover, the user in the coverage area does not need to consider the interval switching when the platform moves, thereby greatly reducing the position update and interval switching caused by the platform swing and rotation, thereby improving system efficiency. Therefore, it can effectively avoid the influence of platform swing and rotation on the system.

The height of the platform is in kilometers. The traditional beamforming method is used, and a twodimensional phased array antenna with beam direction angle $\left(\theta_{0}, \phi_{0}\right)$ is used. 


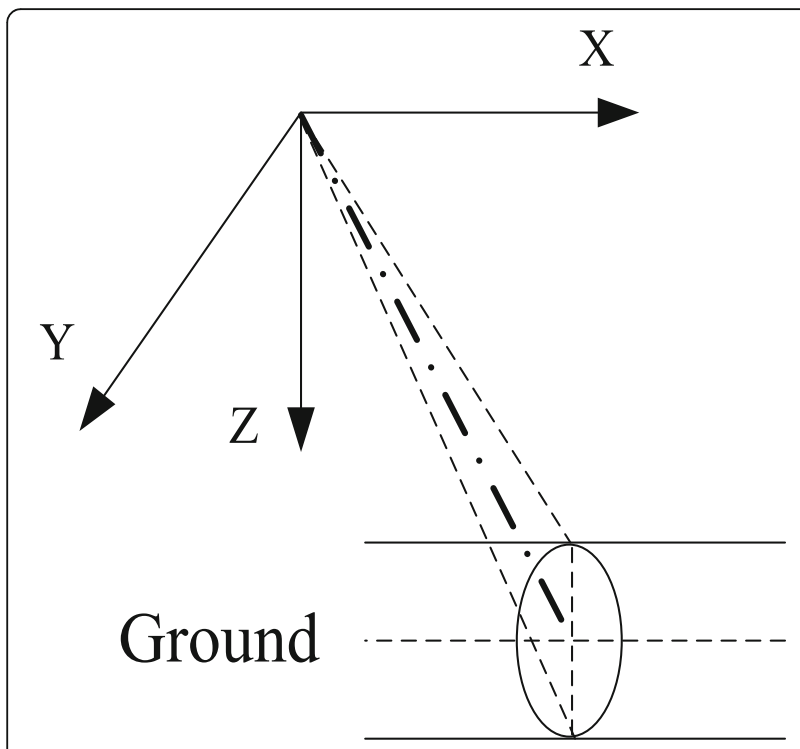

Fig. 1 Schematic diagram of a single banded cell

Communication is possible when the user is in the beam range. Therefore, the setting of beam dwell time and scanning frequency is a crucial factor. The system design must be properly modified to meet the scanning structure of this beam.

Assuming that the beam azimuth is $\phi_{c}$, the overlap angle between two consecutive beams is $\phi_{c v}$, and the angle between the bisectors at the intersection of two consecutive beams is $\phi_{c z}$, as shown in Fig. 3.

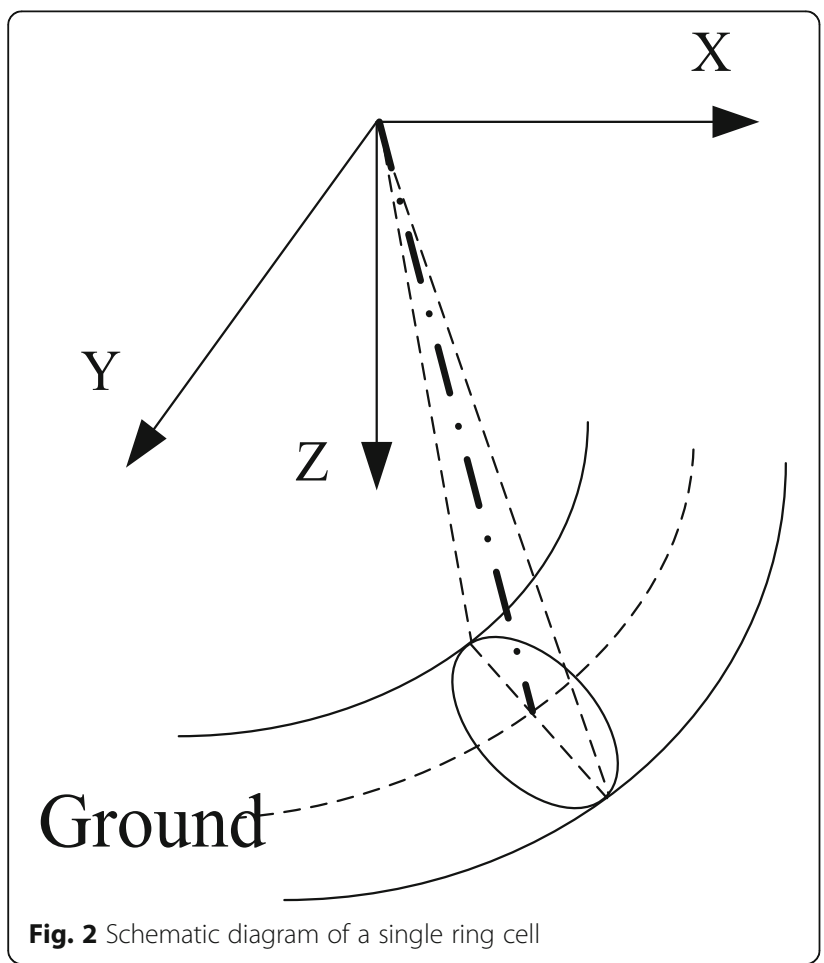

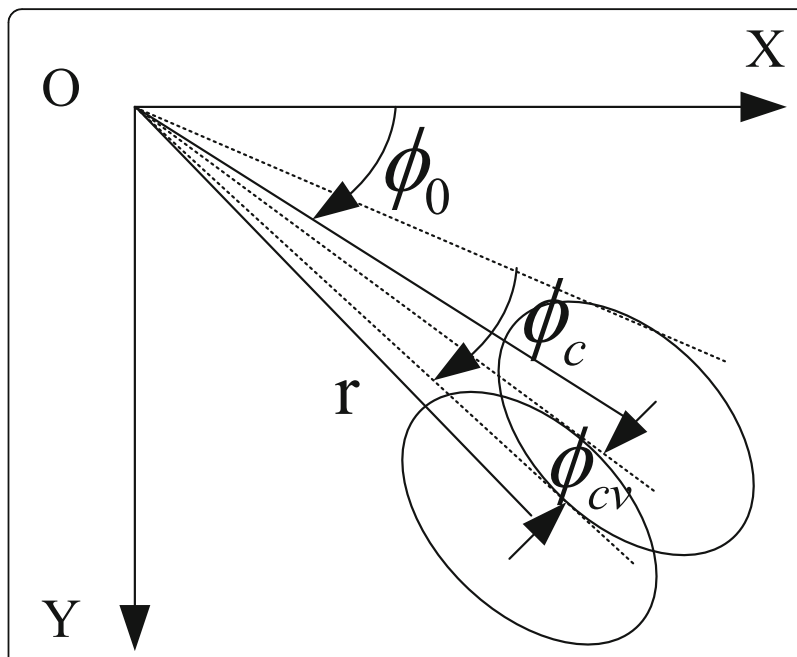

Fig. 3 Sketch map of cell overlap

The ground azimuth $\phi_{c}$ can be approximated as

$$
\phi_{c} \approx 2 \arcsin \left(\sin \left(\frac{B W_{\phi}}{2}\right) \sqrt{1+\cot ^{2} \theta_{0}}\right)
$$

$B W_{\phi}$ is the beamforming half-beam bandwidth in the $\phi_{0}$ plane.

Figure 4 shows the relationship between ground azimuth and antenna dimensions for different antenna elevation angles. As the antenna dimensions increase, the ground azimuth decreases and tends to be equal.

It is assumed that each ring cell is determined by the inner ring radius $r_{1}$, the center radius $r$, and the outer ring radius $r_{2}$, and the ring cell width is $w_{r}$. When $\phi_{0}=$ 0 , the half beam bandwidth is

$$
\begin{aligned}
& B W_{\theta}=\frac{1}{\cos \theta_{0} \sqrt{\theta_{x 0}^{-2} \cos ^{2} \phi_{0}+\theta_{y_{0}}{ }^{-2} \sin ^{2} \phi 0}} \\
& B W_{\phi}=\frac{1}{\sqrt{\theta_{x 0}^{-2} \sin ^{2} \phi_{0}+\theta_{y_{0}}^{-2} \cos ^{2} \phi_{0}}}
\end{aligned}
$$

The uniform linear phased array antenna composed of $N$ similar elements, whose element space is $d$, is equally divided into $M$ subarrays $(1 \leq M \leq N)$, which are recorded as $M \times N$ subarray antenna arrays.

$\theta_{x 0}, \theta_{y 0}$ is the one-dimensional beam bandwidth of the antenna array $M \times N$, and the size is

$$
\theta_{x 0}=\sin ^{-1}\left(\sin \theta_{0}+\frac{0.443 \lambda}{M d_{x}}\right)-\sin ^{-1}\left(\sin \theta_{0}-\frac{0.443 \lambda}{M d_{x}}\right)
$$




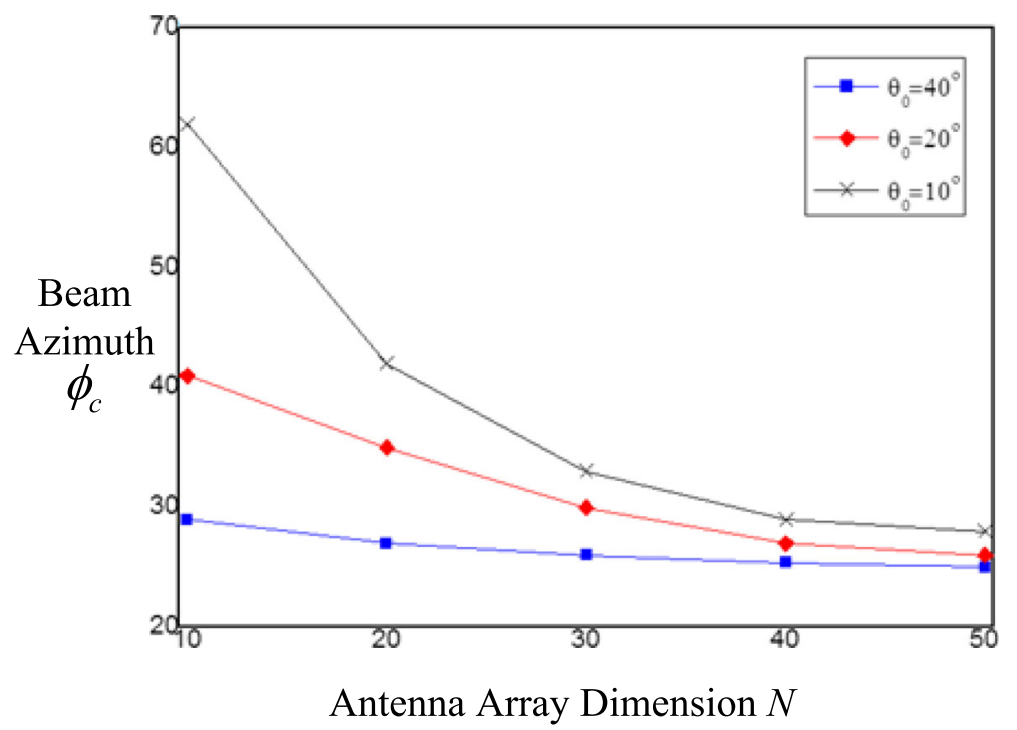

Fig. 4 The relationship of different antenna depression angles $\theta_{0}$ and the beam azimuth $\varphi_{c}$

$$
\theta_{y 0}=\sin ^{-1}\left(\sin \theta_{0}+\frac{0.443 \lambda}{N d_{y}}\right)-\sin ^{-1}\left(\sin \theta_{0}-\frac{0.443 \lambda}{N d_{y}}\right)
$$

It can be seen from Eqs. (13) and (14) that the size of the $M \times N$ antenna array in the $x$-axis and $y$-axis directions determines the beam bandwidth of the antenna. Obviously, when $M, N$ are not equal, the antenna beam size will follow the orientation. The change of angle $\phi_{0}$ would result in a change in the width of the ring cell, which inevitably results in inconsistent size of the ring cell.

In order to keep the size of each ring cell consistent, consider the square antenna of the $M=N$ platform antenna.

For the sake of simplicity of analysis, assuming $d_{x}=$ $d_{y}=0.5 \lambda$, the ring cell width can be expressed as

$$
w_{r}=2 h\left(\frac{\tan \left(\frac{B W_{\theta}}{2}\right)}{\cos ^{2} \theta_{0}-\sin ^{2} \theta_{0} \tan ^{2}\left(\frac{B W_{\theta}}{2}\right)}\right)
$$

The area of the ring cell $A_{r}$ can be expressed as

$$
A_{r}=4 \pi h^{2}\left(\frac{\tan \theta_{0} \tan \left(\frac{B W_{\theta}}{2}\right)}{\cos ^{2} \theta_{0}-\sin ^{2} \theta_{0} \tan ^{2}\left(\frac{B W_{\theta}}{2}\right)}\right)
$$

It can be seen from Eqs. (15) and (16) that $w_{r}$ increases as the depression angle $\theta_{0}$ increases (the elevation angle decreases), and decreases as the dimension of the antenna array increases. Therefore, in order to alleviate the problem that the performance of the communication link is degraded when the ring cell is too wide at low elevation angles, this paper uses a multi-array antenna (increasing the value of $N$ ). The area of the ring cell increases with the increase of the depression angle $\theta_{0}$, and decreases as the antenna array dimension ( $N$ value) increases. By adopting the design of the ring cell, the impact of the platform rotation on the HAPS communication link is effectively suppressed. Figure 5 shows the relationship between the antenna depression angle $\theta_{0}$ and the width of the ring cell $w_{r}$ when the height of the platform is 20 $\mathrm{km}$. It can be seen from Fig. 6 that the area of the ring cell increases as the depression angle $\theta_{0}$ increases, and decreases as the antenna array dimension ( $N$ value) increases.

The multi-layer ring cell scheme is proposed in this paper. The scheme is increasing the inner circle beam width and reducing the outer circle beam width, thereby minimizing the difference between the inner and outer cell link SNRs. At the same time, it also increases the area of the inner circle cell and reduces the area of the outer circle cell. When designing the cell structure, it is also considered to reduce the number of cells by reasonably allocating beams. According to the development of multi-beam antennas in the literature, the number of beams of multi-beam antennas in foreign countries can reach more than 200, while the domestic development is slow. The number of beams currently considered is below 35, an example of a four-layer ring cell with a single platform elevation angle of $5^{\circ}$.

The beam distribution is shown in Figs. 7 and 8. The antenna beam distribution expression is 


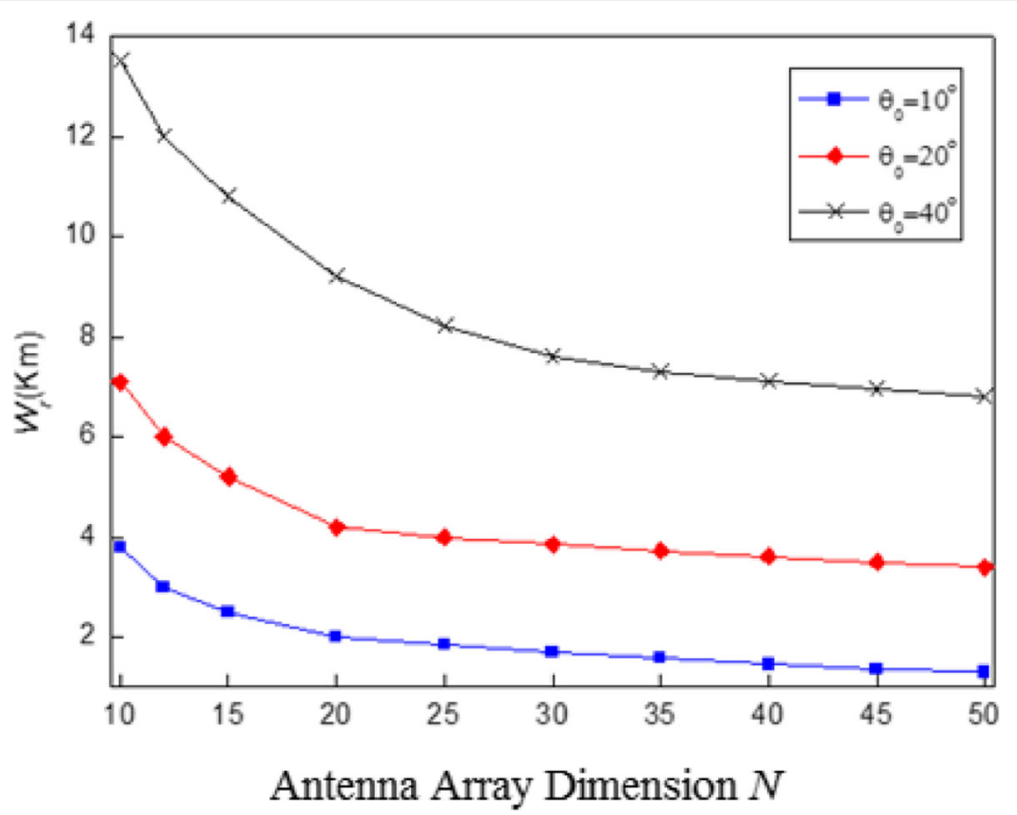

Fig. 5 Relationship between antenna dimensions and ring cell width under different antenna elevation angles $\theta_{0}$

$$
{ }_{1}\left(1_{40}\right)+{ }_{2}\left(9_{20}\right)+{ }_{3}\left(18_{7}\right)+{ }_{4}\left(27_{5}\right)=55
$$

In Eq. (17), the front chaetosema indicates the ring number, the lower chaetosema indicates the half beam width, and the middle number indicates the beam number. For example, ${ }_{1}\left(1_{40}\right)$ means that the first ring (intermediate ring) has a beam with a half beam width of $40^{\circ}$. This solution center uses wide beam coverage with a half beam width of $40^{\circ}$. Because the communication distance is short, the elevation angle is high, and the channel is good, so that communication needs can be

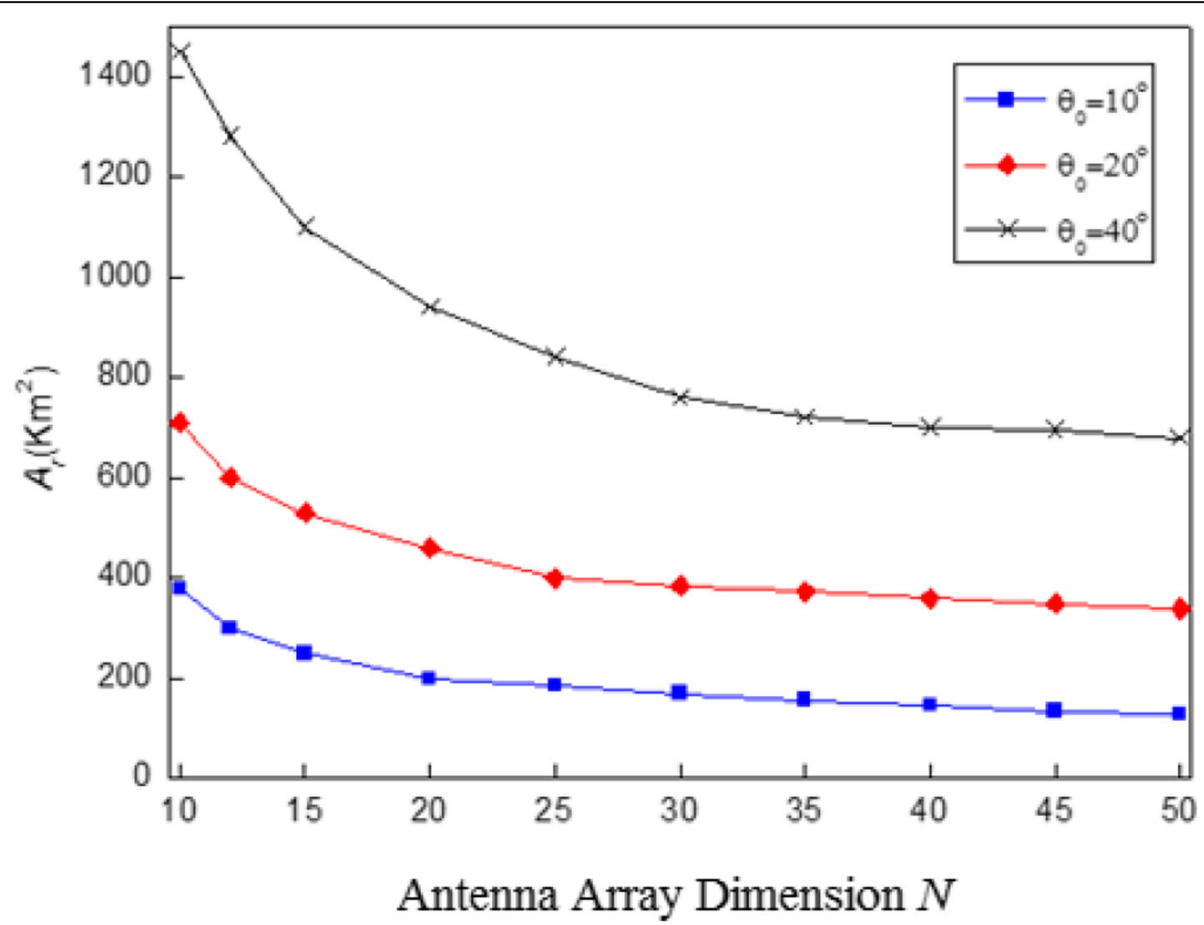

Fig. 6 Relationship between antenna dimensions and annular cell area under different antenna elevation angles $\theta_{0}$ 


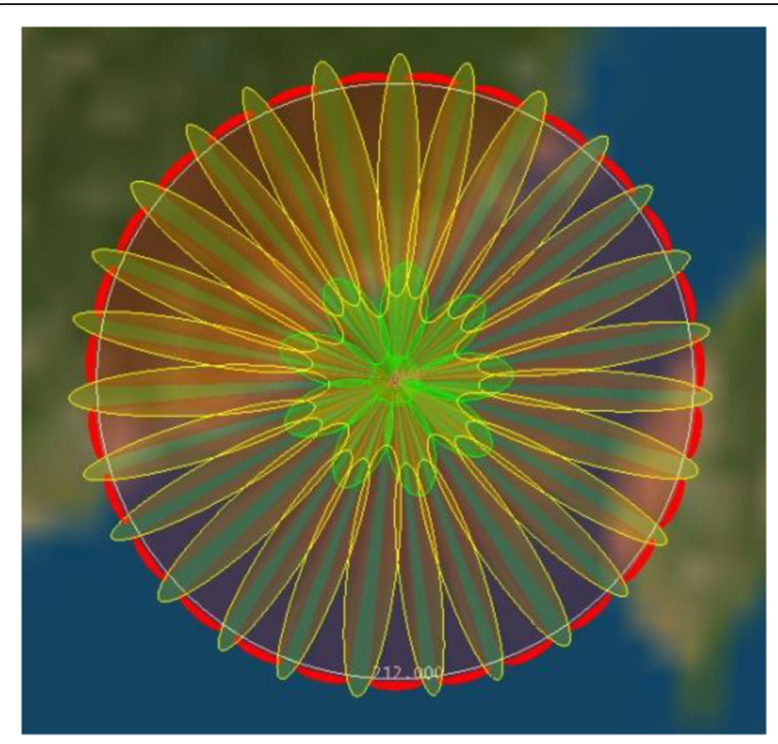

Fig. 7 Schematic diagram of a three-platform four-layer ring cell waveform with a single platform at $5^{\circ}$ elevation angle ( 55 beams)

met. In the edge region, a beam with a half beam width of $5^{\circ}$ is used to compensate for the negative effects of long communication distance and low elevation angle by maximizing the antenna gain.

The link needs to meet the SNR of the communication requirements while leaving a certain margin. However, higher power and G/T values are required for antennas of platforms and user terminals. The downlink has a large margin relative to the uplink SNR.

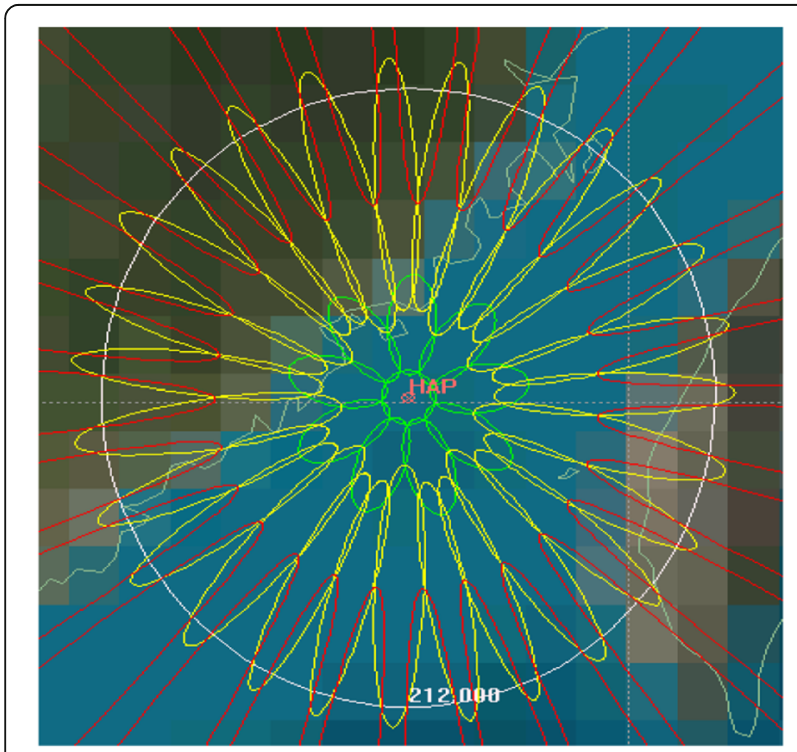

Fig. 8 Schematic diagram of a two-platform four-layer ring cell waveform with a single platform at $5^{\circ}$ elevation angle ( 55 beams)

\section{Results and discussion}

Multi-beam antenna technology has an automatic adaptive beam steering function, which actually concentrates the transmit power in other directions to the main beam direction for directional transmission. Therefore, compared with the conventional antenna, the signal using the multi-beam antenna technology can be transmitted to a much farther distance under the condition of the same transmission power, thereby increasing the coverage. This topic considers the use of an improved elliptical cell scheme: Increasing the inner circle beam width and reducing the outer ring beam width to minimize the difference between the internal and external cell link SNR. At the same time, the area of the inner circle cell is increased, and the area of the outer circle cell is reduced. This study considers reducing the number of cells by reasonably allocating beams, which satisfies both the requirements of coverage link quality and the difficulty of forming antenna beams. This research subject applies the scheme and strategy of improving multi-beam antenna technology in the same system. Under the requirement of cell coverage and partitioning, the related technologies and strategies combining multibeamforming technology and space-time block code technology can obtain high coding gain and diversity gain without sacrificing bandwidth, and effectively improve the wireless systematic capacity.

\section{Abbreviations}

CCl: Co-channel interference; FDD: Frequency division duplex; HAPS: High altitude platform station; ISI: Inter-symbol interference; LCMV: Linear constrained minimum variance; SDMA: Spatial division multiple access; SINR: Signal-to-interference and noise ratio; SNR: Signal-to-noise ratio; TDD: Time division duplex; XPD: Cross-polarization discrimination

\section{Acknowledgements}

The research presented in this paper was supported by the Department of Education of Guangdong Province and Shenzhen Science and Technology Innovation Committee, China

\section{Authors' contributions}

MG is the main writer of this paper. He proposed the main idea, deduced the performance of beamforming, completed the simulation, and analyzed the result. ZW and LW introduced the multi-beam coverage technology. YC, $X C$, and JY simulated the algorithm. BP gave some important suggestions for the establishment of the simulation environment. All authors read and approved the final manuscript.

\section{Funding}

This paper is supported by the Guangdong Province higher vocational colleges \& schools Pearl River scholar funded scheme (2016), the project of Shenzhen Science and Technology Innovation Committee

(JCYJ20170817114522834, JCYJ20160608151239996), Key laboratory of Longgang District (LGKCZSYS2018000028), the national natural science foundation of China (61971081), the science and technology development center of Ministry of Education of China (2017A15009), and Engineering Applications of Artificial Intelligence Technology Laboratory (PT201701) 
Received: 5 July 2019 Accepted: 11 December 2019

Published online: 30 December 2019

\section{References}

1. M. Guan, Z. Wu, Y. Cui, X. Cao, L. Wang, J. Ye, P. Bao, An intelligent wireless channel allocation in HAPS $5 \mathrm{G}$ communication system based on reinforcement learning. EURASIP J. Wirel. Commun. Netw. 138, 1-9 (2019)

2. M. Guan, L. Wang, X. Liu, Joint rate and BER scheduling resource allocation for wireless communication systems. IEEE ACCESS 6, 65697-65704 (2018)

3. J. Li, K.B. Letaief, R.S. Cheng, Z. Cao, Adaptive antenna arrays for co-channel interference cancellation in space-time coded systems. IEEE Vehicular Technology Conference., 38-42 (2011)

4. M.L. McCloud, L.L. Scharf, M.K. Varanasi, Beamforming, diversity, and interference rejection for multiuser communication over fading channels with a receive antenna array. IEEE Trans. Commun. 51(1), 856-861 (2003)

5. L. Xin, Z. Xueyan, J. Min, 5G-based green broadband communication system design with simultaneous wireless information and power transfer. Physical Communication 25, 539-545 (2018)

6. X. Liu, M. Jia, X. Zhang, A novel multichannel Internet of Things based on dynamic spectrum sharing in $5 \mathrm{G}$ communication. IEEE Internet Things J. 6(4), 5962-5970 (2019)

7. X. Liu, X. Zhai, W. Lu, C. Wu, QoS-quarantee resource allocation for multibeam satellite industrial Internet of Things with NOMA. IEEE Transactions on Industrial Informatics 99, 1-10 (2019)

8. A.F.S.N. Naguib, A.R. Calderbank, Space-time coding and signal processing for high-data-rate wireless communications. IEEE Signal Process. Mag. 27(3), 77-91 (2010)

9. F. Zhu, M.S. Lim, Combined beamforming with space-time block coding using double antenna array group. Electron. Lett. 40(13), 1746-1747 (2004)

10. Z. Na, J. Wang, C. Liu, M. Guan, Z. Gao, Join trajectory optimization and communication design for UAV-enabled OFDM networks. Ad Hoc Netw. 98 $1-10(2020)$

11. M. Guan, L. Wang, P. Bao, Adaptive separation of subcarrier for wireless link of satellite communication. Wirel. Pers. Commun. 103(1), 159-166 (2018)

12. M. Guan, L. Wang, Minimum-power constraint-based resource allocation algorithm for wireless communication systems. Journal of Internet Technology 18(3), 245-252 (2017)

13. W.-C. Lee, Y. Shin, H.-C. Kim, Block ordered JBSTBC scheme in highly correlated MIMO channels. Conference Record / IEEE Global Telecommunications Conference., 2079-2083 (2003)

14. M. Guan, L. Wang, L. Chen, Channel allocation for hot spot areas in HAPS communication based on the prediction of mobile user characteristics. Intelligent Automation \& Soft Computing 22(4), 613-62045 (2016)

15. Y.Z. CF et al., Performance comparison of tansmit diversity and beamforming for the downlink of DS-CDMA system. IEEE Trans. On Wireless Communications. 12(2), 320-334 (2013)

16. N. Zhenyu, Y. Wang, L. Xiaotong, X. Junjuan, L. Xin, X.M. Weidang, Subcarrier allocation based Simultaneous Wireless Information and Power Transfer algorithm in $5 \mathrm{G}$ cooperative OFDM communication systems. Physical Communication 29, 164-170 (2018)

17. Z. Na, J. Lv, M. Zhang, B. Peng, M. Xiong, M. Guan, GFDM based wireless powered communication for cooperative relay system. IEEE Access 7 , 50971-50979 (2019)

18. A. Dammann, R. Raulefs, S. Kaiser, Beamforming in combination with spacetime diversity for broadband OFDM systems. IEEE International Conference on Communications., 165-171 (2002)

\section{Publisher's Note}

Springer Nature remains neutral with regard to jurisdictional claims in published maps and institutional affiliations.

\section{Submit your manuscript to a SpringerOpen ${ }^{\circ}$ journal and benefit from:}

- Convenient online submission

- Rigorous peer review

- Open access: articles freely available online

- High visibility within the field

- Retaining the copyright to your article

Submit your next manuscript at $\boldsymbol{\nabla}$ springeropen.com 